\title{
Mass Spectrometry Reveals the Direct Action of a Chemical Chaperone
}

\author{
Joseph Gault, ${ }^{\dagger}$ Danai Lianoudaki, ${ }^{\ddagger}$ Margit Kaldmäe, ${ }^{\ddagger}$ Nina Kronqvist, ${ }^{\S}$ Anna Rising, ${ }^{\S, \otimes}$ Jan Johansson, ${ }^{\S}$
} Bernhard Lohkamp, " Sonia Laín, ${ }^{\ddagger}$ Timothy M. Allison, ${ }^{\perp}$ David P. Lane, ${ }^{*}{ }^{\ddagger}$ Erik G. Marklund, ${ }^{\#}$ and Michael Landreh*,\$(0)

${ }^{\dagger}$ Department of Chemistry, University of Oxford, South Parks Road, Oxford OX1 3QZ, United Kingdom

${ }^{\ddagger}$ Science for Life Laboratory, Department of Microbiology, Tumor and Cell Biology, Karolinska Institutet, Tomtebodavägen 23A, 17165 Stockholm, Sweden

${ }^{\S}$ Division for Neurogeriatrics, Department of Neurobiology, Care Sciences and Society (NVS), Karolinska Institutet, 14183 Huddinge, Sweden

${ }^{\otimes}$ Swedish University of Agricultural Sciences, Dept of Anatomy, Physiology and Biochemistry, Box 7011, 750 07 Uppsala, Sweden "Department of Medical Biochemistry and Biophysics, Karolinska Institutet, Solnavägen 9, 17177 Stockholm, Sweden

${ }^{\perp}$ Biomolecular Interaction Centre and School of Physical and Chemical Sciences, University of Canterbury, Christchurch 8140 , New Zealand

\#Department of Chemistry - BMC, Uppsala University, Box 576, 75123 Uppsala, Sweden

\section{Supporting Information}

ABSTRACT: Despite their fundamental biological importance and therapeutic potential, the interactions between chemical chaperones and proteins remain difficult to capture due to their transient and nonspecific nature. Using a simple mass spectrometric assay, we are able to follow the interactions between proteins and the chemical chaperone trimethylamine- $N$-oxide (TMAO). In this manner, we directly observe that the counteraction of TMAO and the denaturant urea is driven by the exclusion of TMAO from the protein surface, whereas the surfactant lauryl dimethylamine- $N$-oxide cannot be displaced. Our results clearly demonstrate a direct chaperoning mechanism for TMAO, corroborating extensive computational studies, and pave the way for the use of nondenaturing mass spectrometry and related techniques to study chemical chaperones in molecular detail.

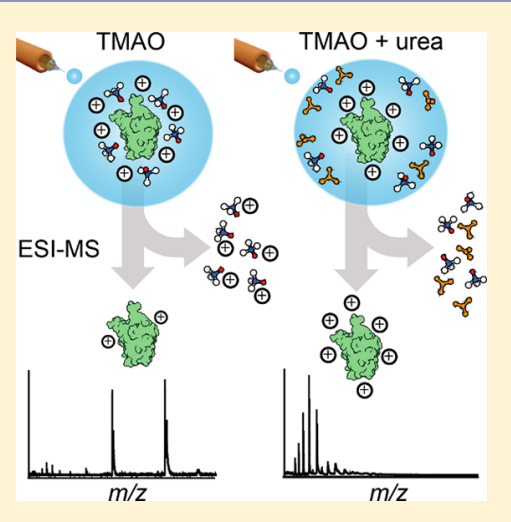

C hemical chaperones are small molecules that protect proteins from denaturation. While some chemical chaperones are used as therapeutic agents targeting specific disease-associated proteins, others exert a broad influence on cellular protein folding. ${ }^{1}$ One of the best-studied examples of the latter type is trimethylamine- $N$-oxide (TMAO), which has a well-established propensity to counteract urea-induced protein unfolding. ${ }^{2,3}$ Its unique properties have been harnessed to stabilize disease-related proteins in vivo. ${ }^{4}$ Owing to the fundamental biophysical role of chemical chaperones, the molecular mechanism underlying their actions has been subject to extensive investigations using experimental and theoretical strategies. ${ }^{5}$ While denaturants such as urea unfold proteins via energetically favorable direct interactions, ${ }^{6}$ the counteracting mechanisms that produce compact protein conformations remain debated, with both indirect and direct mechanisms proposed. For example, TMAO may stabilize proteins by interacting with the protein surface or the denaturant, ${ }^{7,8}$ change the general properties of the bulk solution, ${ }^{9,10}$ or promote compact conformations by inducing an osmophobic effect $^{11}$ that causes the protein to "shy away" from the solvent. ${ }^{12,13}$

Contributing to the ambiguity surrounding the effects of TMAO on protein folding is the fact that most experimental approaches report either solely on the state of the protein (for example, CD $\left.{ }^{14} \mathrm{FRET}^{15}{ }^{\mathrm{DSC}},{ }^{16} \mathrm{NMR}^{17}\right)$ or on the organization of solvent molecules (X-ray scattering). ${ }^{18}$ To date, only molecular dynamics (MD) simulations have been able to provide detailed glimpses of how ternary solutions of urea, TMAO, and model proteins are organized, but these studies have given rise to conflicting interpretations. The prevailing model derived from the simulations suggests that urea is displaced from the protein surface by TMAO, resulting in a transition from unfolded to compact states. ${ }^{12,19-21}$ Yet, two fundamental aspects of this model have largely eluded experimental investigation: Can we detect interactions between

Received: June 11, 2018

Accepted: July 5, 2018

Published: July 5, 2018 
TMAO and proteins and how are these interactions affected by urea?

Nondenaturing mass spectrometry (MS) is well-suited for the study of protein structures and interactions. Using nanoelectrospray ionization (nESI), proteins can be transferred to the gas phase through gentle solvent evaporation from aerosolized microdroplets, resulting in ions with largely intact intramolecular contacts. ${ }^{22}$ Analyses of the protein masses and charges provide insights into their interactions and structures, including the effects of ions and solution additives. ${ }^{23-27}$ The ability of MS to inform about a wide range of interactions raises the possibility to probe potential contacts between TMAO and proteins.

As a starting point, we decided to record nESI-MS spectra of proteins in TMAO-containing solutions and chose the MaSp1 $\mathrm{N}$-terminal domain (NT) because its structure and chemical stability have been extensively characterized in solution and with MS. ${ }^{28-30}$ We found that under gentle ionization conditions, where the solvent is slowly evaporated from the droplets, NT in the presence of 0.1-100 mM TMAO yields well-resolved spectra. The charge state distribution of the desolvated protein is centered around the $6+$ and $7+$ ions, in good agreement with compactly folded NT (Figure 1A). ${ }^{31}$

Intriguingly, we also noticed that when the instrument conditions were altered to promote more efficient ion desolvation the protein signal shifted markedly to the higher $m / z$ region, but only in the presence of TMAO. Raising the voltage of the sample cone in the ion source of the mass spectrometer increases the acceleration of the ions during the final stages of desolvation, resulting in thermal activation. Remarkably, even a moderate increase of the cone voltage significantly reduced the number of charges of the NT ions (Figure 1A). To gain more insights into this process, we titrated NT with increasing TMAO concentrations, recorded spectra over a range of activation energies (cone voltages), and determined the average charge of the protein at each step (Figure 1B). The results clearly show that the charge-reducing effect is dependent on the concentration of TMAO in solution.

In the absence of thermal activation, we observed minor peaks adjacent to the $6+$ charge state of NT corresponding to one and two bound TMAO molecules (Figure S1A). Isolation and dissociation of these complexes in the mass spectrometer showed that the loss of bound TMAO reduces the charge of the protein ion (Figure S1B). This observation confirms that charge reduction is due to the dissociation of TMAO molecules from the protein. However, the magnitude of this effect seems to strongly depend on the desolvation conditions. Under gentle conditions at low cone voltages, TMAO molecules appear to evaporate from the protein with the solvent, leaving the average charge of the NT ions unaffected. Thermal activation at high cone voltages, on the other hand, promotes the dissociation of TMAO as charged species, substantially reducing the protein charge.

On the basis of our observations, we can deduce how TMAO interacts with NT by considering the mechanisms for protein charge reduction through solution additives. ${ }^{32-34}$ It has been proposed that compounds with high basicities interact with positively charged sites on the protein. During the last stages of the ESI process, when the droplets containing the protein are evaporated to dryness, these basic adducts dissociate together with protons from the protein surface. In fact, some MD studies have predicted that TMAO is incorporated into the hydration shell of proteins, forming
(A)

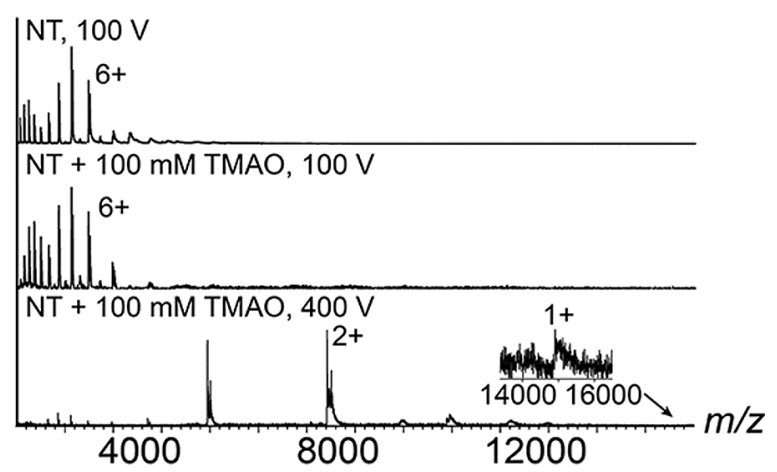

(B)

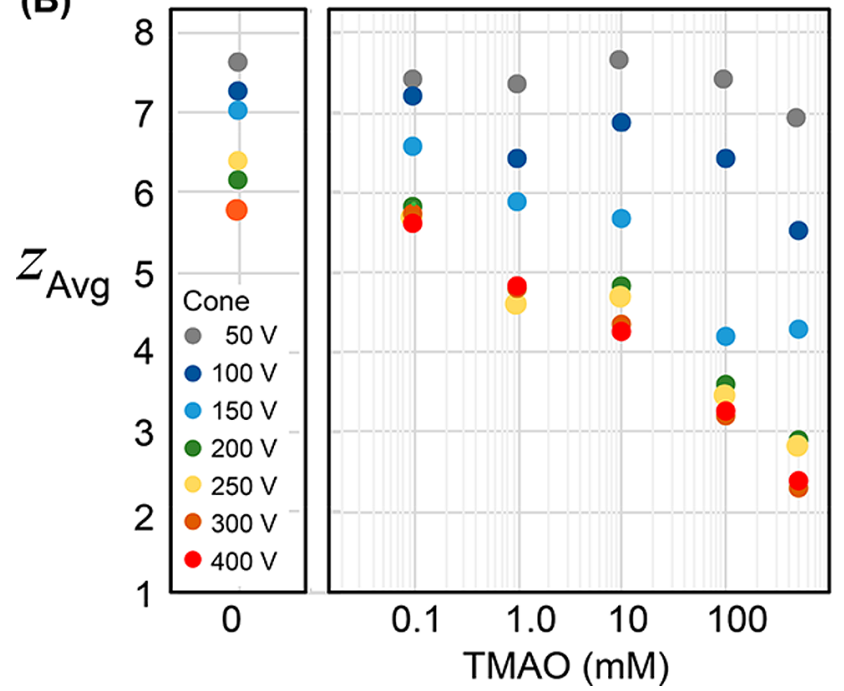

Figure 1. (A) Activation of NT sprayed from ammonium acetate/ TMAO solutions causes significant charge reduction. At a cone voltage of $100 \mathrm{~V}, \mathrm{NT}$ remains centered at around the 7+ charge in the presence of $100 \mathrm{mM}$ TMAO. Increasing the voltage to $400 \mathrm{~V}$ produces predominantly $3+$ and $2+$ ions, and a minor $1+$ ion. (B) Titration of NT with TMAO concentrations from 0.1 to $500 \mathrm{mM}$ gives rise to progressive charge reduction in a concentration- and activation voltage-dependent manner. $Z_{\mathrm{AVG}}$ denotes the protein average charge.

hydrophobic interactions via its methyl groups as well as ionion and ion-hydrogen bonding via its oxygen. ${ }^{12,35,36}$ The observed charge reduction can be explained as the dissociation of bound TMAO molecules from charge-carrying sites on the surface of NT. Our results demonstrate that TMAO readily forms complexes with NT in solution, which can be dissociated during desolvation, indicating a direct interaction between the protein and TMAO.

We now wondered if we could detect how these interactions are affected in ternary solutions of protein, TMAO, and urea. Numerous studies have shown that urea and TMAO have counteracting effects on protein stability. ${ }^{3,8,12,21,35}$ Having established that we can follow the association of NT and TMAO by monitoring the charge reduction during desolvation, we again followed this process in the presence of urea. First, we acquired spectra of NT with urea alone and surprisingly were able to obtain MS data in up to $3 \mathrm{M}$ urea, just on the denaturation threshold for NT. ${ }^{37}$ At this concentration, we detected an additional charge state series centered on the $11+$ ion, which may indicate a more unfolded NT population (Figure S2). Next, we recorded spectra of NT 
in the presence of both urea and TMAO. Strikingly, we observed that in the presence of $3 \mathrm{M}$ urea the charge-reducing effect of TMAO was completely abolished, yielding spectra centered on the $6+$ and $7+$ ions regardless of the ionization conditions (Figure 2A). Furthermore, no NT-TMAO or $\mathrm{NT}$-urea complexes were observed in the presence of urea (Figure S3).

(A)

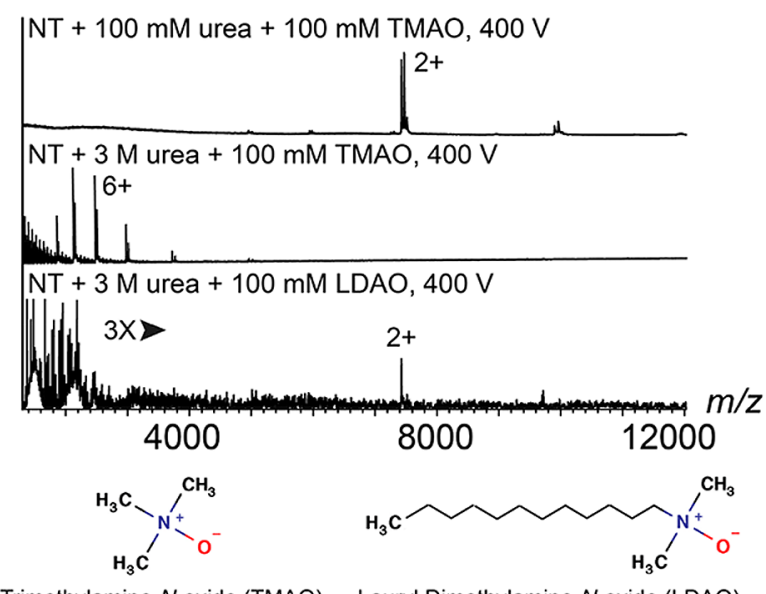

Trimethylamine- $\mathrm{N}$-oxide (TMAO) Lauryl Dimethylamine- $\mathrm{N}$-oxide (LDAO)

(B)

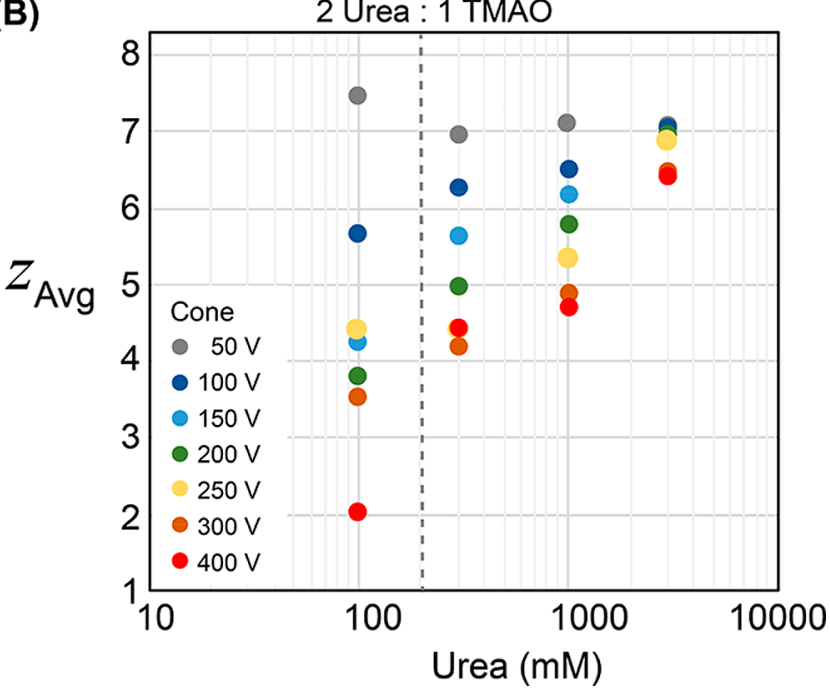

Figure 2. (A) Urea mitigates the charge-reducing effect of TMAO but not that of LDAO. At a urea/TMAO ratio of 1 , urea does not impact TMAO-mediated charge reduction, while at a ratio of 30 , no charge reduction is observed. In the presence of LDAO and urea, we detect detergent clusters below $3000 \mathrm{~m} / z$ and only the $2+$ charge state for NT $(3 \times$ zoom). The structures of TMAO and LDAO are shown below. (B) Charge reduction by $100 \mathrm{mM}$ TMAO is mitigated by urea in a concentration-dependent manner and abolished below a urea/ TMAO ratio of 2 (dashed line). $Z_{\mathrm{AVG}}$ denotes the protein average charge.

We then titrated NT at a fixed TMAO concentration of 100 $\mathrm{mM}$ with urea concentrations ranging from $100 \mathrm{mM}$ to $3 \mathrm{M}$, increased the cone voltage stepwise, and determined the average protein charge in each condition (Figure 2B). At 3 and $1 \mathrm{M}$ urea (urea/TMAO ratios of 30 and 10), only minor charge reductions of 1-2 charges were observed. However, when reducing the urea concentration from 300 to $100 \mathrm{mM}$ (ratios of 3 and 1), the average charge of NT dropped to 4.3 and 2.1, respectively, slightly lower than that with TMAO alone. We repeated the experiment using the C-terminally truncated $36 \mathrm{kDa}$ protein human phosphotyrosine phosphatase $1 \mathrm{~B}$ (Figure S4). At a cone voltage of $400 \mathrm{~V}$, the presence of $500 \mathrm{mM}$ urea did not have a significant impact on protein charge states, while the addition of $50 \mathrm{mM}$ TMAO reduced the average charge from 11.3 to 4.7 . This effect could be reversed by addition of $500 \mathrm{mM}$ urea, suggesting that this behavior is not specific to NT. Our findings are in good agreement with reports that $\sim 2$ is the critical urea/TMAO ratio for TMAOmediated stabilization. ${ }^{13,15}$ Thus, we conclude that urea interferes with the interactions between NT and TMAO in a ratio-dependent manner.

MD studies suggest that urea and TMAO displace each other from the protein surface. ${ }^{12,20,21}$ Cremer and co-workers proposed that TMAO can act as a surfactant for the heterogeneous surfaces of folded proteins. ${ }^{7}$ This type of interaction, we reasoned, would be greatly enhanced if the weakly amphiphilic TMAO was substituted for a highly amphiphilic surfactant that is significantly harder to displace from the protein surface. We therefore used lauryl dimethylamine- $\mathrm{N}$-oxide (LDAO), a detergent that is commonly utilized for solubilization of integral membrane proteins and has been found to interfere with urea-induced denaturation. ${ }^{38}$ In LDAO, one of the methyl groups of TMAO is substituted for an unbranched $\mathrm{C}_{12} \mathrm{H}_{25}$ chain, making it highly amphiphilic (Figure 2A).

Similar to TMAO, titration of NT with LDAO at increasing cone voltages revealed effective charge reduction (Figure S5). The effect appears to occur only above the LDAO critical micelle concentration of 1-2 $\mathrm{mM}$. However, unlike with TMAO, addition of $3 \mathrm{M}$ urea in the presence of $100 \mathrm{mM}$ LDAO did not reverse its charge-reducing effect (Figure 2A). To also evaluate whether LDAO affects urea-induced denaturation in solution, we used tryptophan fluorescence spectroscopy of $\mathrm{NT}^{30}$ In agreement with the MS data, even low concentrations of LDAO were able to prevent the ureainduced shift of the emission maximum for the single tryptophan in NT (Figure S6). LDAO furthermore caused quenching of the fluorescence intensity, suggesting an interaction between detergent and protein. Together, this leads us to conclude that urea, although able to disrupt interactions between TMAO and the protein surface, cannot readily displace the more surface-active detergent LDAO.

Multiple computational studies have suggested antagonistic effects of TMAO and urea on their interactions with proteins. $^{12,20,21}$ Our findings demonstrate directly that excess urea displaces TMAO from protein surfaces. It should be noted that nESI-MS cannot be used to follow TMAO-induced refolding of NT as TMAO concentrations above $500 \mathrm{mM}$ suppress ionization. Yet, urea displaces TMAO even below the concentration necessary for NT unfolding, which suggests that the effect is dependent on the ratio of both compounds rather than a specific concentration. In line with our findings, FRET studies on intrinsically disordered proteins, which are more sensitive to compaction and unfolding than globular proteins, have shown that the counteraction of TMAO and urea is concentration-independent. ${ }^{15}$ Notably, it also points beyond a purely osmophobic mechanism as that would likely not occur at low urea concentrations. The reversion of urea-induced unfolding of globular proteins is therefore likely a macroscopic manifestation of the microscopic displacement mechanism demonstrated here. 
Regarding whether TMAO counteracts urea via a direct or indirect mechanism, our results point to a direct mode: TMAO alone is able to interact directly with the protein surface in the absence of urea, which agrees well with previous MD studies. $^{7,36}$ At increasing urea concentrations, those interactions are disrupted, giving way to direct protein-urea interactions and unfolding ${ }^{19}$ and possibly the formation of TMAO-urea complexes. ${ }^{13}$ Although our data do not report directly whether urea can be displaced by TMAO, they argue in favor of an antagonistic mechanism via exclusion from protein surfaces, as inferred from MD studies. In line with this model, we find that increasing the energetic penalty for dissociating TMAO from the protein by introducing a hydrophobic tail shifts the equilibrium between binding and displacement, favoring attachment to the protein instead. In summary, we provide direct experimental evidence for a competition mechanism between a chemical chaperone and a denaturant that gives rise to antagonistic effects on protein unfolding. Furthermore, in demonstrating the ability of MS to capture chaperone- and denaturant-related effects, this study paves the way for the use of MS and related techniques such as ion mobility spectroscopy to gain new insights into the role of chemical chaperones in protein folding.

\section{ASSOCIATED CONTENT}

\section{S Supporting Information}

The Supporting Information is available free of charge on the ACS Publications website at DOI: 10.1021/acs.jpclett.8b01817.

Experimental methods and Figures S1-S6, showing adduct formation and dissociation results, the effects of the addition of urea and LDAO, nESI-MS spectra of PTP1B, and tryptophan fluorescence of NT in the presence of urea and TMAO (PDF)

\section{AUTHOR INFORMATION}

\section{Corresponding Authors}

*E-mail: David.Lane@ki.se (D.P.L.).

*E-mail: Michael.Landreh@ki.se (M.L.).

\section{ORCID}

Joseph Gault: 0000-0001-7686-2983

Erik G. Marklund: 0000-0002-9804-5009

Michael Landreh: 0000-0002-7958-4074

\section{Author Contributions}

J.G. and M.L. designed the research. D.L. and M.K. performed experiments. N.K., A.R., J.J., and B.L. provided protein. J.G., S.L., T.M.A., D.P.L., E.G.M., and M.L. analyzed and discussed the data. J.G., T.M.A., E.G.M., and M.L. wrote the paper with input from all authors.

\section{Notes}

The authors declare no competing financial interest.

\section{ACKNOWLEDGMENTS}

The authors would like to thank Prof. Dame Carol V. Robinson and Prof. Justin L. P. Benesch, University of Oxford, for encouragement and valuable discussions. M.L. is supported by an Ingvar Carlsson Award from the Swedish Foundation for Strategic Research, a KI faculty-funded Career Position, and a KI-StratNeuro starting grant. J.G. is a Junior Research Fellow of The Queen's College, University of Oxford. E.G.M. holds a Marie Skłodowska-Curie International Career Grant from the
European Commission and the Swedish Research Council (2015-00559). S.L. and D.P.L. acknowledge support from the Swedish Research Council (VR), Cancerfonden, Barncancerfonden, and Karolinska Institutet. M.L. gratefully acknowledges technical support from MS Vision. Special thanks is extended to Meike Bleeksma and Paula Lavfqvist Boqvist (KI) for help with the PTP1B preparations.

\section{REFERENCES}

(1) Lindquist, S. L.; Kelly, J. W. Chemical and biological approaches for adapting proteostasis to ameliorate protein misfolding and aggregation diseases: progress and prognosis. Cold Spring Harbor Perspect. Biol. 2011, 3, a004507.

(2) Wang, A.; Bolen, D. W. A naturally occurring protective system in urea-rich cells: Mechanism of osmolyte protection of proteins against urea denaturation. Biochemistry 1997, 36, 9101-9108.

(3) Yancey, P. H.; Somero, G. N. Counteraction of urea destabilization of protein structure by methylamine osmoregulatory compounds of elasmobranch fishes. Biochem. J. 1979, 183, 317-323.

(4) Brown, C. R.; Hong-Brown, L. Q.; Welch, W. J. Correcting temperature-sensitive protein folding defects. J. Clin. Invest. 1997, 99, $1432-1444$

(5) Canchi, D.; García, A. Cosolvent Effects on Protein Stability. Annu. Rev. Phys. Chem. 2013, 64, 273-293.

(6) Zangi, R.; Zhou, R.; Berne, B. J. Urea's action on hydrophobic interactions. J. Am. Chem. Soc. 2009, 131, 1535-1541.

(7) Liao, Y.-T.; Manson, A. C.; DeLyser, M. R.; Noid, W. G.; Cremer, P. S. Trimethylamine $\mathrm{N}$-oxide stabilizes proteins via a distinct mechanism compared with betaine and glycine. Proc. Natl. Acad. Sci. U. S. A. 2017, 114, 2479-2484.

(8) Ganguly, P.; Boserman, P.; Van Der Vegt, N. F. A.; Shea, J. E. Trimethylamine $\mathrm{N}$-oxide Counteracts Urea Denaturation by Inhibiting Protein-Urea Preferential Interaction. J. Am. Chem. Soc. 2018, 140, 483-492.

(9) Breydo, L.; Sales, A. E.; Ferreira, L.; Fedotoff, O.; Shevelyova, M. P.; Permyakov, S. E.; Kroeck, K. G.; Permyakov, E. A.; Zaslavsky, B. Y.; Uversky, V. N. Effects of osmolytes on protein-solvent interactions in crowded environment: Analyzing the effect of TMAO on proteins in crowded solutions. Arch. Biochem. Biophys. 2015, 570, 66-74.

(10) Ma, J.; Pazos, I. M.; Gai, F. Microscopic insights into the protein-stabilizing effect of trimethylamine $\mathrm{N}$-oxide (TMAO). Proc. Natl. Acad. Sci. U. S. A. 2014, 111, 8476-8481.

(11) Bolen, D. W.; Baskakov, I. V. The osmophobic effect: Natural selection of a thermodynamic force in protein folding. J. Mol. Biol. 2001, 310, 955-963.

(12) Smolin, N.; Voloshin, V. P.; Anikeenko, A. V.; Geiger, A.; Winter, R.; Medvedev, N. N. TMAO and urea in the hydration shell of the protein SNase. Phys. Chem. Chem. Phys. 2017, 19, 6345-6357.

(13) Meersman, F.; Bowron, D.; Soper, A. K.; Koch, M. H. J. Counteraction of urea by trimethylamine $\mathrm{N}$-oxide is due to direct interaction. Biophys. J. 2009, 97, 2559-2566.

(14) Henkels, C. H.; Oas, T. G. Thermodynamic characterization of the osmolyte- and ligand-folded states of Bacillus subtilis ribonuclease P protein. Biochemistry 2005, 44, 13014-13026.

(15) Ferreon, A. C. M.; Moosa, M. M.; Gambin, Y.; Deniz, A. A. Counteracting chemical chaperone effects on the single-molecule -synuclein structural landscape. Proc. Natl. Acad. Sci. U. S. A. 2012, 109, 17826-17831.

(16) Bruździak, P.; Panuszko, A.; Stangret, J. Influence of osmolytes on protein and water structure: a step to understanding the mechanism of protein stabilization. J. Phys. Chem. B 2013, 117, 11502-11508.

(17) Doan-Nguyen, V.; Loria, J. P. The effects of cosolutes on protein dynamics: the reversal of denaturant-induced protein fluctuations by trimethylamine N-oxide. Protein Sci. 2007, 16, 20-29.

(18) Sahle, C. J.; Schroer, M. A.; Juurinen, I.; Niskanen, J. Influence of TMAO and urea on the structure of water studied by inelastic Xray scattering. Phys. Chem. Chem. Phys. 2016, 18, 16518-16526. 
(19) Bennion, B. J.; Daggett, V. The molecular basis for the chemical denaturation of proteins by urea. Proc. Natl. Acad. Sci. U. S. A. 2003, 100, 5142-5147.

(20) Canchi, D. R.; Jayasimha, P.; Rau, D. C.; Makhatadze, G. I.; Garcia, A. E. Molecular mechanism for the preferential exclusion of TMAO from protein surfaces. J. Phys. Chem. B 2012, 116, 1209512104.

(21) Ganguly, P.; Hajari, T.; Shea, J. E.; Van Der Vegt, N. F. A. Mutual exclusion of urea and trimethylamine $\mathrm{N}$-oxide from amino acids in mixed solvent environment. J. Phys. Chem. Lett. 2015, 6, 581585.

(22) Konermann, L.; Ahadi, E.; Rodriguez, A. D.; Vahidi, S. Unraveling the mechanism of electrospray ionization. Anal. Chem. 2013, 85, 2-9.

(23) Hall, Z.; Robinson, C. V. Do charge state signatures guarantee protein conformations? J. Am. Soc. Mass Spectrom. 2012, 23, 11611168.

(24) Lössl, P.; van de Waterbeemd, M.; Heck, A. J. The diverse and expanding role of mass spectrometry in structural and molecular biology. EMBO J. 2016, 35, 2634-2657.

(25) Sterling, H. J.; Prell, J. S.; Cassou, C. A.; Williams, E. R. Protein conformation and supercharging with DMSO from aqueous solution. J. Am. Soc. Mass Spectrom. 2011, 22, 1178-1186.

(26) Han, L.; Hyung, S. J.; Mayers, J. J. S.; Ruotolo, B. T. Bound anions differentially stabilize multiprotein complexes in the absence of bulk solvent. J. Am. Chem. Soc. 2011, 133, 11358-11367.

(27) Warnke, S.; Von Helden, G.; Pagel, K. Protein structure in the gas phase: The influence of side-chain microsolvation. J. Am. Chem. Soc. 2013, 135, 1177-1180.

(28) Landreh, M.; Askarieh, G.; Nordling, K.; Hedhammar, M.; Rising, A.; Casals, C.; Astorga-Wells, J.; Alvelius, G.; Knight, S. D.; Johansson, J.; et al. A pH-Dependent Dimer Lock in Spider Silk Protein. J. Mol. Biol. 2010, 404, 328-336.

(29) Jaudzems, K.; Askarieh, G.; Landreh, M.; Nordling, K.; Hedhammar, M.; Jörnvall, H.; Rising, A.; Knight, S. D.; Johansson, $\mathrm{J}$. pH-dependent dimerization of spider silk $\mathrm{N}$-terminal domain requires relocation of a wedged tryptophan side chain. J. Mol. Biol. 2012, 422, 477-487.

(30) Kronqvist, N.; Otikovs, M.; Chmyrov, V.; Chen, G.; Andersson, M.; Nordling, K.; Landreh, M.; Sarr, M.; Jörnvall, H.; Wennmalm, S.; et al. Sequential $\mathrm{pH}$-driven dimerization and stabilization of the $\mathrm{N}$ terminal domain enables rapid spider silk formation. Nat. Commun. 2014, 5, 3254.

(31) Landreh, M.; Andersson, M.; Marklund, E. G.; Jia, Q.; Meng, Q.; Johansson, J.; Robinson, C. V.; Rising, A. Mass spectrometry captures structural intermediates in protein fiber self-assembly. Chem. Commun. 2017, 53, 3319-3322.

(32) Catalina, M. I.; van den Heuvel, R. H. H.; van Duijn, E.; Heck, A. J. R. Decharging of globular proteins and protein complexes in electrospray. Chem. - Eur. J. 2005, 11, 960-968.

(33) Hopper, J. T. S.; Sokratous, K.; Oldham, N. J. Charge state and adduct reduction in electrospray ionization-mass spectrometry using solvent vapor exposure. Anal. Biochem. 2012, 421, 788-790.

(34) Susa, A. C.; Mortensen, D. N.; Williams, E. R. Effects of cations on protein and peptide charging in electrospray ionization from aqueous solutions. J. Am. Soc. Mass Spectrom. 2014, 25, 918-927.

(35) Bennion, B. J.; Daggett, V. Counteraction of urea-induced protein denaturation by trimethylamine $\mathrm{N}$-oxide: A chemical chaperone at atomic resolution. Proc. Natl. Acad. Sci. U. S. A. 2004, 101, 6433-6438.

(36) Cho, S. S.; Reddy, G.; Straub, J. E.; Thirumalai, D. Entropic stabilization of proteins by TMAO. J. Phys. Chem. B 2011, 115, 13401-13407.

(37) Andersson, M.; Chen, G.; Otikovs, M.; Landreh, M.; Nordling, K.; Kronqvist, N.; Westermark, P.; Jörnvall, H.; Knight, S.; Ridderstråle, Y.; et al. Carbonic Anhydrase Generates $\mathrm{CO} 2$ and $\mathrm{H}+$ That Drive Spider Silk Formation Via Opposite Effects on the Terminal Domains. PLoS Biol. 2014, 12, e1001921.
(38) Maurya, S. R.; Mahalakshmi, R. Cysteine residues impact the stability and micelle interaction dynamics of the human mitochondrial $\beta$-barrel anion channel hVDAC-2. PLoS One 2014, 9, e92183. 